\title{
Prediction of life stress on athletes' burnout: the dual role of perceived stress
}

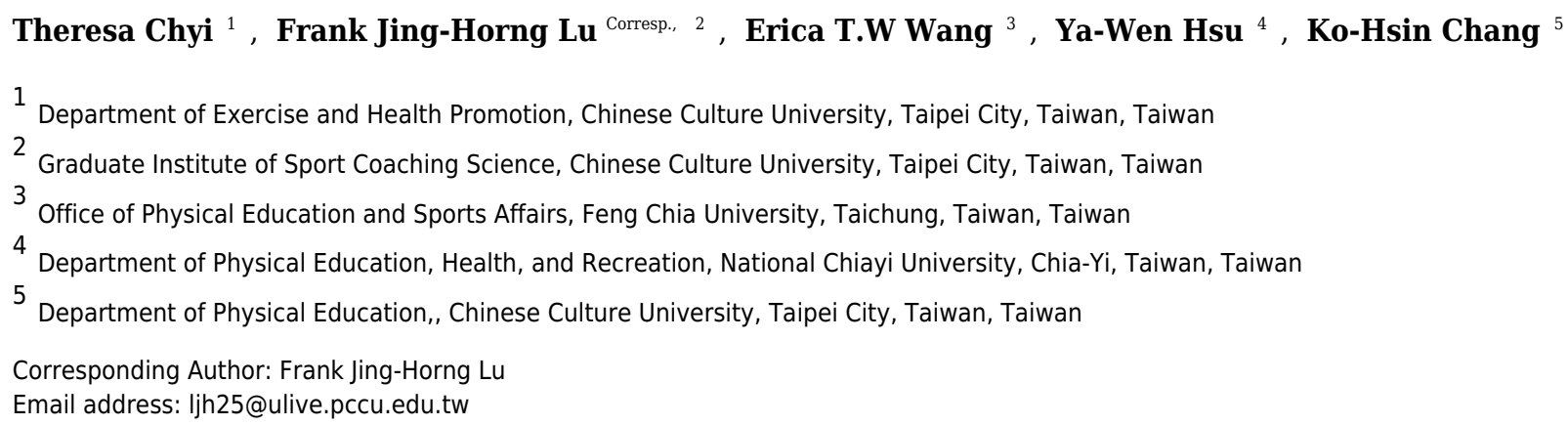

Although many studies adopted Smith (1986) cognitive-affective model of athletic burnout in examining stress-burnout relationship, very few studies examined the mediating/moderating role of perceived stress on the stress-burnout relationship. We sampled 195 college student-athletes and assessed their life stress, perceived stress, and burnout. Correlation analyses found all study variables correlated. Two separate hierarchical regression analyses found that the "distress" component of perceived stress mediated athletes' two types of life stress-burnout relationship but "counter-stress" component of perceived stress moderated athletes' general-life stress-burnout relationship. We concluded that interweaving relationships among athletes' life stress, perceived stress, and burnout are not straightforward. Future research should consider the nature of athletes life stress, and dual role of perceived stress in examining its' association with related psychological responses in athletic settings. 


3

Prediction of Life Stress on Athletes' Burnout :

The Dual Role of Perceived Stress

\author{
Theresa Chyi ${ }^{1}$, Frank J.H. Lu ${ }^{1}$, Erica T.W. Wang ${ }^{2}$, Ya-Wen Hsu ${ }^{3}$ \\ \& Ko-Hsin Chang ${ }^{1}$ \\ ${ }^{1}$ Chinese Culture University \\ ${ }^{2}$ Feng Chia University \\ ${ }^{3}$ National Chia-Yi University
}

Correspondence concerning this article should be addressed to Frank J.H. Lu

Institute: Graduate Institute of Sport Coaching Science, Chinese Culture University, Address: \#55, Hua-Kang Road, Yang-Ming Shang, Taipei, TAIWAN, 11114.

Phone: 1-886-933-029265

Fax: 1-886-3-3280596

E-mail: 1jh25@ulive.pccu.edu.tw

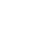

9

0

1

2

\title{
Theresa Chyi ${ }^{1}$
}

Department of Exercise and Health Promotion, Chinese Culture University

Authors Information 
40 Address: \#55, Hua-Kang Road, Yang-Ming-Shan, Taipei, TAIWAN. 11114

41 Tel: 1-886-2-28610511 ext .43831 (office)

42 E-mail: tcc@ulive.pccu.edu.tw

Frank J. H. Lu ${ }^{* 1}$

45 Graduate Institute of Sport Coaching Science, Chinese Culture University

46 Address: \# 55, Hua-Kang Road, Yang-Ming-Shan, Taipei, TAIWAN. 11114

47 Tel: 1-886-2-28610511 ext .43226 (office)

48 E-mail: ljh25@ulive.pccu.edu.tw

50 Erica T.W. Wang ${ }^{2}$

51 Office of Physical Education and Sports Affairs, Feng-Chia University

52 Address: \#100, Wenhwa Rd., Seatwen, Taichung, Taiwan 40724

53 Tel: 1-886-4-24517250 ext. 5985 598

54 E-mail: tingwen830@hotmail.com

\section{Ya-Wen Hsu ${ }^{3}$}

57 Department of Physical Education, Health, and Recreation, National Chia-Yi University

58 Address: \#300 Syue-fu Rd., Chiayi, Taiwan

59 Tel: TEL: +886-5-2717000 FAX: +886-5-2717095

60 E-mail: tgieva@gmail.com

61 
Prediction of Life Stress on Athletes' Burnout :

The Dual Role of Perceived Stress

in examining stress-burnout relationship, very few studies examined the mediating/moderating

role of perceived stress on the stress-burnout relationship. We sampled 195 college student-

athletes and assessed their life stress, perceived stress, and burnout. Correlation analyses found

all study variables correlated. Two separate hierarchical regression analyses found that the

“distress" component of perceived stress mediated athletes' two types of life stress-burnout relationship but "counter-stress" component of perceived stress moderated athletes' general-life stress-burnout relationship. We concluded that interweaving relationships among athletes' life stress, perceived stress, and burnout are not straightforward. Future research should consider the nature of athletes life stress, and dual role of perceived stress in examining its' association with related psychological responses in athletic settings. 


\section{Introduction}

Playing sports in college as student-athletes is never an easy task for young adults. The college student-athletes are ordinary students; they have to cope with college-related demands such as preparing course works, passing exams, arranging campus life, taking care of personal health, and handling interpersonal relationships. Besides, they are competitive athletes; they have to engage in athletically-related tasks such as participating in vigorous training/competitions, building coaches/teammates relationship, preventing/taking care of injuries, and meeting performance demands. In addition, if they are freshmen or transfer students they have to face many life adjustment issues, such as moving to new residence, adapting new school/social systems, or building new social relationships (Etzel, 2009; Huffman, 2014; Lu, Hsu, Chan, Cheen, \& Kao, 2012). For these reasons, student-athletes in the campus are very vulnerable to stress.

Despite stress is an inevitable part of life, research indicated that chronic stress is detrimental to physical and mental health. Many studies indicated that overly exposed to daily life stress increasing heart disease (Steptoe \& Kivimaki, 2012), suffering from gastrointestinal ulcers (Ali \& Harty, 2009), elevating the possibility of cancer (Valko, Rhodes, Moncol, Izakovic, \& Mazur, 2006), causing asthma (Wright, Rodriguez, \& Cohen, 1998), and increasing hyperglycemia (Bosarge \& Kerby, 2013). As to mental problems, research found high in stress is associated with depression (Risch et al., 2009), increasing hopelessness and suicide ideation (Davidson, Wingate, Rasmussen, \& Slish, 2009; Glick, Stillman, Reardon, \& Ritvo, 2012), lowering well-being (DiBartolo \& Shaffer, 2002), decreasing performance (Humphrey, Yow, \& Bowden, 2000), increasing eating disorder (Epel, Lapidus, McEwen, \& Brownell, 2001), and developing burnout (Francisco, Arceb, Vílcheza, \& Valesc, 2016; Gustafsson et al., 2012; Lu et al., 2016). 

by researchers and is closely associated with stress. Athlete burnout is defined as “....an athlete's reaction to chronic stress... which is characterized as feeling physically and psychologically exhausted from the demands of training and competing, perceive a reduced sense of accomplishment, and experience sport devaluation in which they engage (Raedeke \& Smith, 2001, p.283 ). In explaining the process of burnout, Smith (1986) proposed a four-stage theory of athletic burnout which contended that burnout is a reaction to chronic stress comprising situational, cognitive, physiological, and behavioral components. The first stage starts from perceived situational demands including performance demands, conflicts between training and personal schedule, overload training, expectations and pressures from others. The second stage termed cognitive appraisal by which athletes' interpret these demands; particularly individuals' cognitive appraisal of the balance between challenges and resources, and potential consequences. When athletes perceived demands surpass personal resources and consequences will be severe, the process goes to the third stage of physiological and psychological responses such as anxiety, tension, insomnia, and illness. The forth stage is athlete burnout which is characterized by rigid and inappropriate behavior, decreased performance and withdrawal from activity.

Many studies had adopted Smith (1986) framework in examining the stress-burnout relationship. Generally, past research supports the association between stress and athlete burnout. For example, in an effort to examine the antecedent and consequence of athlete burnout, Francisco et al., (2016) investigated 453 Spanish athletes and measured their perceived stress, depression, and burnout. Results found perceived stress accounted for $43 \%$ of the variance of burnout, and perceived stress and burnout jointly accounted for $50 \%$ of the variance of depression. Similarly, by using a qualitative approach, Gustafsson and colleagues (Gustafsson, Hassmen, Kenta, \& Johansson, 2008) interviewed 11 Swedish athletes about the sources of burnout. They found multiple demands such as "too much sport," " lack of recovery" and "high 
expectations" were considered as primary causes of burnout. Further, in a search of sources and characteristics of athlete burnout, Cresswell, and Eklund (2006) interviewed 15 New Zealand professional rugby players found many negative experiences such as injury, perceptions of the team environment and training loadings associated with burnout. Recently, Gustafsson and colleagues (Gustafsson \& Skoog, 2012) examined whether athletes high in optimism negatively predicted burnout, they measured participants' optimism, perceived stress, and burnout. Results found a mediating effect of perceived stress on the optimism-burnout relationship. Moreover, Gustafsson and colleagues (Gustafsson, Skoog, Podlog, Lundqvist, \& Wagsson, 2013) investigated the relationships among hope, perceived stress, and found perceived stress mediated the hope-burnout relationship.

Despite these efforts in examining the stress-burnout relationship, very few studies include both life stressors and global perceived stress in Smith (1986) cognitive-affective model of athletic burnout. According to Smith (1986), athletes encounter many situational demands from life, training, and competition. These demands are life stressors including engaging in heavy training, meeting performance demands, preventing/taking care of the injury, and building/maintaining an interpersonal relationship. Another type of stress is the global perception of the stress. That is, how athletes perceived these stressors are stressful. Smith (1986) contends that when athletes encounter life stressors they would engage in the cognitive appraisal. To measure this type of global perception of stress, most researchers (e.g., Gustafsson et al., 2012; 2013; Raedeke \& Smith, 2004 ) used Cohen and colleagues' (Cohen, Kamarack, \& Mermelstein, 1983) Perceived Stress Scale (PSS) to assess athletes' perceived stress. Generally, they used a composite score of PSS by reversing all the positive items of PSS and added them to all negative items. Although this approach is simple and straight forward, it omitted lots of information of how perceived stress influence athletes’ psychological responses. According to Cohen and colleagues (1983) perceived stress includes both positive and negative components (i.e., counter 
stress and perceived distress). The "counter stress" represents one's confidence in how he/she can cope any challenge/disturbance that one encounters in life. In contrast, "perceived distress" refers to one's perception of how life's situations are uncontrollable, unexpected, overloading and make one feels distressing and annoying.

Under such consideration, when examining stress-burnout relationship if researchers include two type of stress (i.e., life stress and perceived stress) and differentiate the positive/negative components of perceived stress on the life stress-burnout relationship, they would be able to explore more knowledge about the interweaving relationships among athletes' life stress, perceived stress, and burnout. Especially, recent research on the psychometric properties of PSS (e.g., Barbosa-Leiker et al., 201; Chiu et al., 2016) confirmed that PSS comprises two factors as Cohen et al., (1983) suggested. As to life stress, Lu et al., (2012) developed a sport-specific life stress scale which categorized athletes' life stress into two categories- general-life stress and sport-life stress. Further, empirical studies showed individuals' counter stress cognition such as self-efficacy moderated family caregivers' behavioral problems and burnout relationship (Romero-Morno et al., 2011), or moderated workers' stress appraisal-quality of life relationship (Prati, Pietrantoni, \& Cicognani, 2010), and moderated the relationship between cancer patients' treatment information and well-being (Namkoong et al., 2010).

In contrast, the empirical studies have found perceived distress mediated the relationship between individual personality (e.g., hope and optimism) and burnout relationship as earlier mentioned works by Gustafsson et al., (2012; 2013). In non-sport settings, it was found when nursing students perceived more stress from teaching and learning and other organizational demands they perceived high distress, which in turn led to burnout. Thus, perceived stress mediated the demands-burnout relationship. Moreover, in a large-scale investigation (Behavioral Risk Factor Surveillance System) with a total of 85,130 participants which examined the relationship among perceived discrimination, psychological distress, and smoking status. Results 
found regardless of race/ethnicity, psychological distress mediated the discrimination-smoking association (Pumell et al., 2012).

Unfortunately, to best of our knowledge, no researchers attempted to adopt such consideration in examining the interweaving relationships among two type of life stress, two type of perceived stress and burnout. By using Smith (1986) model as a guiding framework, it is considered that negative component of perceived stress (i.e., perceived distress) would play a mediating role in the two types of life stress-burnout relationship because perceived distress stands in the middle between environmental challenges and athlete burnout. As Baron and Kenny (1986, p.1176) suggested, a mediator is a third variable that explains how external physical events take on internal psychological significance. In contrast, it is considered that positive component of perceived stress (i.e., counter stress) would play a moderating role in the two types of life stress-burnout relationship because counter stress represents athletes' cognitive appraisals that they can handle stress. According to Baron and Kenny's (1986, p.1174), the moderator is the third variable that affects the direction/strength of independent/predictor variable and dependent/criterion variable.

\section{Purposes and hypotheses}

Building on the above literature, the purpose of this study was to examine the relationships among athletes' two types of life stress, the two components of perceived stress, and burnout; and examine the mediating/moderating role of perceived stress on the life stress-burnout relationship. We hypothesized that athletes' two types of life stress, perceived stress (i.e., perceived distress and counter stress), and burnout would be significantly correlated. Further, "perceived distress" would mediate the two types of life stress-burnout relationship but "counter stress" would moderate two types of life stress-burnout relationship. 


\section{Participants}

Participants in this study were 195 athletes (male $=138$, female $=57$ ) with average ages of $19.89(\mathrm{SD}=1.34)$ years recruited from 2 sports colleges and 4 universities in Taiwan. They had been participating in intercollegiate sports such as basketball, volleyball, and baseball with $7.56 \pm$ 2.83 years of sports experiences. They averagely trained 3.54 hours per day $(S D=1.32)$ and 5.02 days per week.

Measurements \& Procedure

Prior to data collection, we gained an approval from the Institutional Review Board of a hospital ethical committee. Then, we contacted athletes through the permissions of coaches and administrators. In an appointed date we arrived their training venues by the introduction of their coaches. Then, we briefly introduced ourselves and informed participants the purpose of the research, confidentiality, and anonymity of their participation. Those who interested in this study then signed informed consent and completed a survey package including the demographic questionnaire, the life stress, perceived stress and burnout measures. It took approximately 20 minutes to complete. The questionnaire administration was either before or after each team's training session. The measures as follow:

Demographic questionnaire: We used a demographic questionnaire to collect participants' age, gender, types of sports, daily training hours, training frequency per week, and years of athletic experiences.

Athlete Burnout Questionnaire (ABQ)

Developed by Raedeke and Smith (2001), the ABQ was used to assess participants' athletes' burnout experiences. The ABQ has three subscales namely: (a) reduced the sense of athletic accomplishment (RA); (b) perceived emotional and physical exhaustion (E), and (c) devaluation of sports participation (D). Participants identify their athletic burnout experiences using a six-point Likert scale that ranged from 1 (never) to 6 (always). Sample question for 
emotional/physical exhaustion is "I feel so tired from my training that I have trouble finding energy to do another thing"; for reduced sense of accomplishment is "I'm accomplishing many worthwhile things in sport"; for sport devaluation is "The effort I spend in sport would be better spent doing other things." The higher the number as participants identified as the higher the degree of burnout of the sport. The internal consistency of the present study was $.85, .86$, and .63. In this research, we used a composite score by adding three subscales together.

\section{College Student-Athletes'Life Stress Scale (CSALSS)}

We used 24-item CSALSS (Lu et al., 2012) to assess participants' perceptions of their daily life stress classified as general-life and sport-specific stress. The questionnaire asked questions such as "I am annoyed with my coach's bias against me," or "I worry about my unstable competitive performance." There are eight factors in the 24-item CSALSS including: (a) sports injury, (b) performance demand, (c) coach relationships, (d) training adaptation, (e) interpersonal relationships, (f) romantic relationships, (g) family relationships, and (h) academic requirements.

Participants indicated the frequency of the specific life event on a six-point Likert scale ranging from 1 (Never) to 6 (Always). Cronbach's $\alpha$ of these factors ranged from .70 to .87 and the reliability for all items was .93 in this study, indicating that the measure was reliable. According to $\mathrm{Lu}$ and colleagues ( $\mathrm{Lu}$ et al., 2012), college student-athletes life stress can be categorized as sport-specific life stress (i.e., sports injury, performance demand, coach relationships, training adaptation) and general-life stress (i.e., interpersonal relationships, romantic relationships, family relationships, academic requirements.) We used the composite score of sport-specific life stress and general-life stress for statistical analyses.

The Perceive Stress Scale (PSS)

Developed by Cohen et al., (1983), the PSS measure is used to assess one's perception of the degree of a given situation in life is stressful. We used a 2-factor 10-item PSS for our study (Chiu et al., 2016). Chiu et al., (2016) reported that 2-factor 10-item PSS with appropriate 
psychometric properties. The sample question of "perceived distress" is (e.g., how often have you felt upset because of something that happened unexpectedly?), and sample question of "counter stress" is (e.g., How often have you felt confident about your ability to handle personal problems?). According to Chiu et al., (2016) items 1,2, 3, 6, 9, 10 represent perceived distress; items 4, 5, 7,8 represent counter stress. Participant rated their experiences of stress by answering items with a 5 -point Likert scale $(0=$ never, $1=$ almost never, $2=$ sometimes, $3=$ fairly often, $4=$ very often). We used a composite score of perceived distress and counter stress by adding all 6/4 items together.

Statistical Analyses

Firstly, the descriptive statistical analysis examined the properties of the collected data, which including skewness, kurtosis, means and standard deviations. Also, we used Pearson product-moment correlation analysis to examine the correlations of all variables.

\section{Examination of Mediation}

For mediating effect, we used a simple regression to examine whether the independent variable predicts mediator; mediating variable predicts dependent variable, and independent variable predicts the dependent variable. This analysis was conducted as a prerequisite analysis for testing mediating effects (Baron \& Kenny, 1986, p. 1176). According to Baron and Kenny (1986), to examine mediation effect, the following conditions should be met in our study: (a) two types of life stress should be able to account for variance in perceived distress; (b) perceived distress should be able to account for variance in burnout; and (c) two types of life stress should be able to account for variance in burnout. If all three conditions were met, the subsequent mediating of perceived stress on the relationship between two types of life stress and burnout were further analyzed. To examine the main effects, two types of life stress (i.e., sport-specific and general-life stress) were entered into the regression in the first step. Perceived distress was entered in the second step. Two types of life stress and perceived distress were entered in the third 
step. The final test of mediation was to examine whether two types of life stress would still predict burnout when perceived distress was controlled. Finally, a Sobel test $(Z \geqq 1.96, p<.05)$ was used to examine the significance of mediating effect.

\section{Examination of Moderation}

To examine moderating effect, we used hierarchical regression analysis to examine the unique and joint contributions of two types of life stress and counter stress in predicting athlete burnout. The control variable (i.e., sex) was entered into the regression first. In the second and third steps, we examined the main effects of two types of life stress and counter stress on burnout. Finally, the full model with interaction effects of two types of life stress and counter stress were tested. Based on procedures recommended by Cohen and colleagues (Cohen, Cohen, West, Aiken, 2002) we graphed all significant interactions to show the relationship between counter stress and burnout using data of 1 standard deviation above and below the mean for two types of life stress.

Results

Preliminary Analysis

Descriptive statistics found skewness ranged from -.01 to .36 and kurtosis ranged from -.15 to -.34 , indicating that study variables did not exceed \pm 2 , and all variables were in an acceptable range of normality (Hair, Black, Babin, Anderson, \& Tatham, 2006). In addition, no outliers were found. Pearson correlation test revealed all variables were positively correlated, the coefficient between .23 to .65 as table 1 indicated. Athlete burnout had a higher correlation with sportspecific life stress than general-life stress and perceived distress. But burnout negatively correlated with counter stress. Further, according to Barron and Kenny's (1986) suggestion of the 
312 prerequisite of mediation effect, a simple regression analysis showed that sport-specific stress and

313 general-life stress positively predicted perceived distress $(\beta=.49, p<.00 ; \beta=.34, p<.00)$ and

314 burnout $(\beta=.50, p<.00 ; \beta=.33, p<.00)$. Also, perceived distress positively predicted burnout

$(\beta=.37, \mathrm{p}<.00)$ as table 2 indicated. Therefore, it is suitable for subsequent mediation analysis.

(insert table 1 and table 2 about here)

\section{Mediating effects of perceived distress on life stress-burnout relationship}

We used hierarchical regression analyses to examine mediation effects. As table 3 (left) shown when perceived distress was controlled in the first step, general-life stress predicted burnout $\left(\mathrm{R}^{2}=.11, \mathrm{~F}(1,194)=23.77\right.$. $)$ However, when perceived stress and general-life stress simultaneously entered in the model 2 the prediction of general-life stress on burnout was significantly less after controlling perceived distress ( $\beta$ from .33 to .23 ). Sobel test found the mediating effect was significant (Sobel 's $Z=3.84, p<.05$ ). Similarly, as table 3 (right) illustrated when perceived distress was controlled in the first step, sport-specific life stress predicted burnout $\left(\mathrm{R}^{2}=.25, \mathrm{~F}(1,194)=65.30\right)$. However, when sport-specific life stress and perceived distress simultaneously entered in the model 2 the prediction of sport-specific life stress on burnout was significantly less after controlling perceived distress ( $\beta$ from .50 to .42). Sobel test found the mediating effect was significant (Sobel 's $Z=4.60, p<.05$ ).

\section{Moderating effects of counter stress on life stress-burnout relationship}

The predictive value of life stress and perceived stress on burnout is illustrated in Table 4.

The main effects of two types of life stress and counter stress were significant. However, only the interaction between general-life stress and counter stress significantly predicted burnout; the 
interaction uniquely accounted for $3 \%$ of the variance. Based on procedures recommended by

Cohen and colleagues the graph of the interaction illustrated that for participants with high counter stress ( 1 standard deviation above the mean) their burnout was lower than those with low counter stress (1 standard deviation below the mean) (Figure 1). The full model accounted for $19.0 \%$ of burnout. However, the interaction between sport-specific life stress and counter stress did not significantly predict burnout.

(insert table 4 and figure 1 about here)

\section{Theoretical contributions/implications}

Discussion

In considering that stress plays an important role in athlete burnout, we adopted Smith (1986) cognitive-affective model of athletic burnout and reexamined the triangular relationships among athletes' life stress, perceived stress, and burnout. Results found all study variables were correlated, and perceived stress plays a dual role in mediating and moderating the life stressburnout relationship. The preliminary results provide several implications as follow:

First, to differentiate the nature and role of stress in the stress-burnout relationship adds our knowledge that both life stress and perceived stress play a different role in athlete burnout. The global perception of stress can be a mediator and moderator in the life stress-burnout relationship. The mediating effect of perceived distress supported past research that this type of stress mediated the relationship between hope and burnout (Gustafsson et al., 2013), optimism and burnout (Gustafsson et al., 2012), and coping source of burnout (e.g., Raedeke \& Smith, 2004). The moderating effect of counter stress also supported that individuals' beliefs that they can handle stress moderated the job demand-burnout relationship (Salanova, Peiro, Schaufeli, 2002), athletes life stress-burnout relationship (Lu et al., 2016), or soldiers' stressor-strain relationship (Jex, Bliese, Buzzell, \& Primeau, 2001).

Further, the finding of mediating and moderating effects of perceived stress added our knowledge of how life stress and perceived stress influence athletes' burnout responses. For mediating effect of perceived distress on the life stress-burnout relationship, it was found both 
"general-life" stress and "sport-specific" life stress and burnout relationships were all mediated by perceived distress. However, in the moderating condition, the only general-life stress-burnout relationship has been moderated by counter stress. The reason for such distinction was unknown. However, we inferred that it might be because our participants were sampled from Division-I athletes. Research indicated that experienced athletes have been taught to use all types of coping, such as increasing efforts, suppressing competing activity, active coping, and seeking social support, for stressful sports situations (Crocker \& Graham, 1995; Pensgaard \& Urshin, 1998). Therefore, the relationship between sport-specific life stress and burnout can't be moderated by counter stress. However, such explanation was just referred from literature. Future studies should empirically examine the relationships among athletes' sport-specific life stress, counter stress, and burnout.

Further, the prediction of life stress on athlete burnout supported that many life stressors associated with burnout (Cresswell \& Eklund, 2006; Franciscoa, et al., 2016; Gustafsson, et al., 2008; Lu et al., 2016). Specifically, this study found sport-specific life stress had higher correlation than general-life stress with burnout. This implied that athletes perceived high in sport-specific life stress are disadvantageous to burnout. Our results prompt coaches, sports administrators, parents, and sports professionals pay attention to athletes' sport-specific life stress because it may influence athletes' well-being in sports.

The athlete burnout is the core issue of the present study. Our results found athlete burnout not only associated with life stress but also mediated/moderated by different type of perceived stress. The dual role of perceived stress on the life stress-burnout relationship informs us that athletes' cognitive appraisal will influence how athletes react to life stressors. According to Lazarus $(1984 ; 1993)$, any event that has been evaluated as stressful is because individuals perceived they are lack of coping resources and abilities. If they perceived they are confident to handle these challenges they would not feel stressful. Also, if they perceived that they have many 
resources to cope with stressors they do not feel stressors are threatening. We suggest future study may adopt this dual role perspective of perceived stress in other areas such as how perceived stress influences athletic injury (Andersen \& Williams, 1988), doping (Hodge, Hargreaves, Gerrard, \& Longsdale, 2013), substance abuse (Grossbard, Hummer, Labrie, Pederon, \& Neighbors, 2009), and eating disorder (Dockendorff, Petrie, Greenleaf, \& Martin, 2012 ).

\section{Limitations and Future Suggestions}

Several methodological and interpretive issues need to be discussed. First, although we found there is a moderating/mediating effect of perceived stress on the life stress-burnout relationship. Because the present study is a cross-sectional study it does not warrant cause-andeffect. Future study may adopt a longitudinal design to observe how athletes' life stress influences stress appraisals, and how perceived stress subsequently influences life stress-burnout relationship. Second, our sample was all recruited from Taiwan and Division-I college studentathletes whether our results can be generalized to other culture, or athletes with different levels such as professional athletes or younger athletes, need to be further examined. Third, in this study, we only measured participants' life stress. Whether another type of life stressors (e.g., organizational stressor) predicted athletes' burnout should be further examined (Arnold, Fletcher, \& Daniels, 2013). Moreover, although we adopted Smith (1986) cognitive-affective model of athletic burnout in examining stress-burnout relationship we did not include psychological/physiological responses which are conceptualized in the third stage. Future study may include these variables in the research model and examine how they are correlated. 
414 This study has shown interweaving relationships of life stresses, perceived stress, and burnout

415 in college student-athletes. The negative component of perceived stress played as a mediator both

416 in general-life and sport-life stress-burnout relationship. On the other hand, the positive

417 component of perceived stress played as a moderator between general-life stress-burnout but not

418 in sport-specific life stress condition. In order to promote total health and wellness of student-

419 athlete, we suggest sports administrators, coaches, and parents should work together to reduce

420 athletes' stress via effective life management programs. By doing so it can build a healthy athletic

421 community and promote athletes' psychological well-being.

422

\section{Acknowledgements}

The authors would like to extend their appreciations toward Ministry of Science and

Technology of Taiwan for its research grant MOST 104-2410-H-179-009

\section{References}

Ali, T., \& Harty, R. F. (2009). Stress-Induced Ulcer Bleeding in Critically Ill Patients. Gastroenterology Clinics of North America, 38, 245-265, DOI: 10.1016/j.gtc.2009.03.002

Anderson, M. B., \& Willians, J. M. (1988). A model of stress and athletic injury: Prediction and prevention. Journal of Sport \& Exercise Psychology, 10, 294-306.

Arnold, R., Fletcher, D., \& Daniels, K. (2013). Development and validation of the Organizational Stressor Indicator for Sport Performers (OSI-SP). Journal of Sport and Exercise Psychology, 35(2), 180-196.

Barron, R. M. \& Kenny, D. A. (1986). The moderator-mediator variable distinction in social psychological research: conceptual, strategic, and statistical considerations. Journal of Personality and Social Psychology, 51, 1173-1182.

Bosarge, P. L., \& Kerby, J. D. (2013). Stress-induced hyperglycemia. Advances in Surgery, 47, 287-297. http://dx.doi.org/10.1016/j.yasu.2013.03.002. 
Chiu, Lu, Lin, Nien, Hsu, \& Liu, (2016). Psychometric Properties of the Perceived Stress Scale (PSS): Measurement Invariance Between Athletes and Non-athletes and Construct Validity. Peer J. DOI 10.7717

Cohen, S., Kamarack, T., \& Mermelstein, R. (1983). A global measure of perceived stress. Journal of Health and Social Behavior, 24, 385-396.

Cohen J, Cohen P, West, S.G., Aiken L.S. (2002). Applied Multiple Regression/Correlation Analysis for the Behavioral Sciences. Mahwah, NJ: Lawrence Erlbaum Associates.

Cresswell, S., \& Eklund, R.C., (2006). The nature of player burnout in rugby: key characteristics and attributions. Journal of Applied Sport Psychology, 18, 219-239.

Crocker, P., \& Graham, T.R. ( 1995). Coping by competitive athletes with performance stress: gender differences and relationships with affect. The Sport Psychologist, 9,325-338.

Davidson, C. L., L. R. Wingate, K. A. Rasmussen and M. L. Slish. (2009). “Hope as a Predictor of Interpersonal Suicide Risk." Suicide and Life-Threatening Behavior, 39(5):499-507. doi: $10.1521 /$ suli.2009.39.5.499

DiBartolo, P.M., \& Shaffer, C. (2002). A Comparison of Female College Athletes and Nonathletes: Eating Disorder Symptomatology and Psychological Well-Being, Journal of Sport and Exercise Psychology, 24(1), 33-41.

Dockendorff, S.A., Petrie, T.A., Greenleaf, C.A., \& Martin, S. (2012 ). Intuitive Eating Scale: An Examination Among Early Adolescents, Journal of Counseling Psychology, 59( 4), 604 611. DOI: 10.1037/a0029962.

Epel, E., Lapidus, R., McEwen, B., \& Brownell, K. (2001). Stress may add bite to appetite in women: a laboratory study of stress-induced cortisol and eating behavior, Psychoneuroendocrinology, 26, 37-49.

Etzel, E. F. (2009). Counseling Student Athletes: Issues and interventions. Morgantown, WV: Fitness Information Inc. 
464 Francisco, C. Arceb, C., Vílcheza, M., \& Valesc, A. (2016).Antecedents and consequences of burnout in athletes: Perceived stress and depression. International Journal of Clinical and Health Psychology, 16, 239-246.

Glick, I.D., Stillman, M.A., Reardon, C.L., \& Ritvo, E.C. (2012). Managing psychiatric issues in elite athletes, The Journal of Clinical Psychology, 73(5):640-644. DOI: 10.4088/JCP.11r07381

Grossbard, J., Hummer, J., Labrie, E., Pederon, E., \& Neighbors, C. (2009). Is Substance Use a Team Sport? Attraction to Team, Perceived Norms, and Alcohol and Marijuana Use Among Male and Female Intercollegiate Athletes, Journal of Applied Sport Psychology, 21: 247261, DOI: 10.1080/10413200903019145

Gustafsson, H., Hassmen, P., Kenta, G., \& Johansson, M. (2008). A qualitative analysis of burnout in elite Swedish athletes. Psychology of Sport and Exercise, 9, 800-816. http://dx.doi.org/10.1016/j.psychsport.2007.11.004

Gustafsson, H. \& Skoog, T. (2012). The mediational role of perceived stress in the relation between optimism and burnout in competitive athletes. Anxiety, Stress, and Coping, 25(2), $183-199$.

Gustafsson, H., Skoog, T., Podlog, Lundquist, Wagnasson, S. (2013). Hope and athlete burnout: Stress and affect as mediators. Psychology of Sport and Exercise, 14, 640-649.

Hair, J. F., Jr., Black, W. C., Babin, B. J., Anderson, R. E., \& Tatham, R. L. (2006). Multivariate data analysis (6th ed.). Upper Saddle River, NJ: Prentice-Hall

Hodge, K., Hargreaves, E. A., Gerrard, D., \& Longsdale, C. (2013). Psychological Mechanisms Underlying Doping Attitudes in Sport: Motivation and Moral Disengagement, Journal of Sport \& Exercise Psychology, 35(4), 419-432. https://doi.org/10.1123/jsep.35.4.419

Huffman, L. T. (2014). Examining Perceived Life Stress Factors among Intercollegiate Athletes: A Holistic Perspective. Unpublished dissertation, University of Tennessee, Knoxville. 
489 490 491 492 493 494 495 496 497 498

Humphrey, J.H., Yow, D.A., \& Bowden, W.W. (2000). Stress in college athletics: Causes, consequences, coping. Binghamton, NY: The Haworth Half-Court Press.

Jex, S.M., Bliese, P. D., Buzzell, S., \& Primeau, J. (2001). The impact of self-efficacy on stressstrain relations: Coping style as an explanatory mechanism. Journal of Applied Psychology, 86 (3), 401-409.

Lazarus, R.S., \& Folkman, S. (1984). Stress, appraisal, and coping. New York: Springer.

Lazarus, R.S., (1993). From psychological stress to emotions: A history of changing outlooks. Annual Review of Psychology, 44, 1-21.

Lu, F. J. H., Hsu, Y. W., Chan, Y. S., Cheen, J. R., \& Kao, K. T. (2012). Assessing college studentathletes' life stress: Initial measurement development and validation. Measurement in Physical Education and Exercise Science, 16(4), 254-267.

Lu, F.J.H., Lee, W.P., Chang, Y.K., Chou, C.C., Hsu, Y.W., Lin, J.H., \& Gill, D.L. (2016). Interaction of athletes' resilience and coaches' social support on the stress-burnout relationship: A conjunctive moderation perspective, Psychology of Sport and Exercise 22, 202-209.

Namkoong, K., Shah, D. V., Han, J.Y. Kim, Yoo, W., \& Fan, D.,... Gustafsond, D.H., (2010). Expression and reception of treatment information in breast cancer support groups: How self-efficacy moderates effects on emotional well-being. Patient Educ Couns. 81S1: S41S47. doi:10.1016/j.pec.2010.09.009

Pensgaard, A. M., \& Ursin, H. (1998). Stress, control, and coping in elite athletes. Scandinavian Journal of Medicine and Science in Sports, 8, 183-189.

Prati, G., Pietrantoni, L., \& Cicognani, E. (2010). Self-efficacy moderates the relationship between stress appraisal and quality of life among rescue workers. Anxiety, Stress, and Coping, 23 (4), 463-470. DOI: 10.1080/10615800903431699 
513 Pumell, J. Q., Peppone, L.J., Alcaraz, K., McQuee, A., Guido, J.J., \& Carroll, J...Morrow, G.R.,

514 (2012). Perceived discrimination, psychological distress, and current smoking status: Results

515 from the Behavioral Risk Factor Surveillance System Reaction to Race Module, 2004-2008.

516 American Journal of Public Health, 102(5), 844-651.

517 Raedeke, T. D., \& Smith, A. L. (2001). Development and preliminary validation of a measure of

518 athlete burnout measure. Journal of Sport and Exercise Psychology, 23, 281-306.

519 Raedeke, T. D., \& Smith, A. L. (2004). Coping resources and athlete burnout: An examination of stress mediated and moderation hypothesis. Journal of Sport and Exercise Psychology, 26, 525-541.

Risch, N., Herrell, R., Lehner, T., Liang, K., Eaves, L., Hoh, J., Griem, A., Kovacs, M., Ott, J. \& Merikangas, K. R. (2009). Interaction Between the Serotonin Transporter Gene (5HTTLPR), Stressful Life Events, and Risk of Depression A Meta-analysis. JAMA, 301, (23), 2462-2471.

Romero-Morno, R., Losada, A., Mausbach, B.T., Marquez-Gonzalez, M., Patterson, T.L., \& Lopez, J. (2011). Analysis of the moderating effect of self-efficacy domains in different points of the dementia caregiving process. Aging and Mental Health, 15 (2), 221-231. DOI: $10.1080 / 13607863.2010 .505231$

Salanova, M., Peiro, J.M., \& Schaufeli, W. B. (2002). Self-efficacy specificity and burnout among information technology workers: An extension of the job demand-control model. European Journal of Work and Organizational Psychology, 11(1), 1-25.

Smith, R. E. (1986). Toward a cognitive-affective model of athletic burnout. Journal of Sport Psychology, 8, 36-50.

Steptoe, A. \& Kivimaki, M (2012). Stress and cardiovascular disease, Nature Reviews Cardiology 9, 360-370,| doi:10.1038/nrcardio.2012.45 
537 Valko, M. Rhodes, C. J., Moncol, J. Izakovic, M., \& Mazur, M. (2006). Free radicals, metals, and 538 antioxidants in oxidative stress-induced cancer. Chemico-Biological Interactions, 160, $1-$ $539 \quad 40$.

540 Vogel, D. L., \& Wei, M., (2005). Adult attachment and help-seeking intent: The mediating roles 541 of psychological distress and perceived social support. Journal of Counseling Psychology, 52 (3), 347-357. DOI: 10.1037/0022-0167.52.3.347

543 Wright, R. J., Rodriguez, M., \& Cohen, S. (1998). Review of psychosocial stress and asthma: an integrated biopsychosocial approach, Thorax, 53, 1066-1074. 


\section{Table $\mathbf{1}$ (on next page)}

correlation matrix and descriptive statistics for study variables

correlation matrix and descriptive statistics for study variables 
Table 1 Correlation matrix and descriptive statistics for study variables

\begin{tabular}{lccccc} 
& 1 & 2 & 3 & 4 & 5 \\
\hline 1. ABQ & $.90^{\mathrm{a}}$ & $.33^{* *}$ & $.50^{* *}$ & $.37^{* *}$ & $-.29^{* *}$ \\
CSALSS & & & & & \\
2. general-life stress & & $.88^{\mathrm{a}}$ & $.65^{* *}$ & $.34^{* *}$ & -.11 \\
3. sport-life stress & & & $.88^{\mathrm{a}}$ & $.49^{* *}$ & $-.23^{* *}$ \\
PSS & & & & & \\
4. distress & & & & $.78^{\mathrm{a}}$ & -.13 \\
5. counter stress & & & & & $.67^{\mathrm{a}}$ \\
Mean & 3.03 & 2.56 & 3.08 & 2.02 & 2.03 \\
SD & .86 & .06 & .06 & .67 & .70 \\
Skewness & -.28 & -.35 & -.30 & -.16 & -.55 \\
Kurtosis & .22 & .37 & -.02 & .10 & .16 \\
\hline
\end{tabular}

Note. ${ }^{\text {a }} \mathrm{ABQ}$ : athlete burnout questionnaire ; CSALSS: college student-athlete life stress scale ; ${ }^{\mathrm{b}}$ Cronbach $\alpha$ for each subscale is displayed on the diagonal; $* p<.05, * * p<.01$. 


\section{Table 2 (on next page)}

Simple regression of life stress and perceived distress on burnout

simple regress of life stress and perceived stress on burnout 
Table 2 Simple regression of life stress and perceived distress on burnout.

\begin{tabular}{ccccc}
\hline \multirow{2}{*}{ Variables } & \multicolumn{2}{c}{ PSS-distress } & \multicolumn{2}{c}{ Burnout } \\
\cline { 2 - 5 } & $\beta$ & $\Delta R^{2}$ & $\beta$ & $\Delta R^{2}$ \\
\hline Regression 1 & & & & \\
Sport stress & $.49^{* *}$ & $.24^{* *}$ &. & \\
General stress & $.34^{* *}$ & $.11^{* *}$ & & \\
Regression 2 & & & & $.25^{* *}$ \\
Sport stress & & & $.50^{* *}$ & $.11^{* *}$ \\
General stress & & & $.33^{* *}$ & $.14^{* *}$ \\
PSS-distress & & & $.37^{* *}$ &
\end{tabular}

Note. ${ }^{\mathrm{a}}$ Dependent variable is PSS-distress; ${ }^{\mathrm{b}}$ dependent variable is Burnout;

${ }^{\mathrm{c}}$ significant ${ }^{*} p<.05 .{ }^{* *} p<.01$. 


\section{Table 3(on next page)}

Mediating effects of PSS-distress on the life stress-burnout relationship

mediating effects of PSS-distress on the life stress-burnout relationship 
Table 3 Mediating effects of PSS-distress on the life stress-burnout relationship

\begin{tabular}{|c|c|c|c|c|c|c|c|c|c|}
\hline & \multicolumn{4}{|c|}{ Burnout } & & \multicolumn{4}{|c|}{ Burnout } \\
\hline & \multicolumn{2}{|c|}{ Step 1} & \multicolumn{2}{|c|}{ Step 2} & & \multicolumn{2}{|c|}{ Step 1} & \multicolumn{2}{|c|}{ Step 2} \\
\hline & $\mathrm{B}$ & $\beta$ & $\mathrm{B}$ & $\beta$ & & $\mathrm{B}$ & $\beta$ & $\mathrm{B}$ & $\beta$ \\
\hline Constant & $2.21 * *$ & & $1.70 * *$ & & Constant & $1.50 * *$ & & $1.31 * *$ & \\
\hline Life Stress-G & $.32 * *$ & .33 & $.22 * *$ & .23 & Life Stress-S & $.50 * *$ & .50 & $.42 * *$ & .42 \\
\hline PSS-distress & & & $.38 * *$ & .29 & PSS-distress & & & $.22 *$ & .17 \\
\hline$R^{2}$ & .11 & & .19 & & $R^{2}$ & .25 & & .27 & \\
\hline Adjusted $R^{2}$ & .11 & & .18 & & Adjusted $R^{2}$ & .25 & & .27 & \\
\hline Changed in $R^{2}$ & & & .08 & & Changed in $R^{2}$ & & & .02 & \\
\hline Sobel 's Z & & & $3.84 *$ & & Sobel 's Z & & & $0 *$ & \\
\hline
\end{tabular}

Note. Life Stress-G: general-life stress; Life Stress-S: sport-specific life stress

$* p<.05, * * p<.01$. 


\section{Table 4(on next page)}

summary results of the moderating effects

summary results of the moderating effect 
Table 4 Summary results of the moderating effects

\begin{tabular}{|c|c|c|c|c|c|c|}
\hline & \multicolumn{3}{|c|}{ LS-G x PSS-Counter } & \multicolumn{3}{|c|}{ LS-S x PSS-Counter } \\
\hline & Predictor & $R^{2}$ & $\beta$ & Predictor & $R^{2}$ & $\beta$ \\
\hline \multirow[t]{3}{*}{ Step 1} & & $.12 * *$ & & & $.26^{* *}$ & \\
\hline & Sex & & .10 & Sex & & .09 \\
\hline & LS-G & & $.35^{* *}$ & LS-S & & $.51 * *$ \\
\hline \multirow[t]{4}{*}{ Step2 } & & $.17 * *$ & & & $.29 * *$ & \\
\hline & Sex & & .05 & Sex & & .05 \\
\hline & LS-G & & $.31^{* *}$ & LS-S & & $.47 * *$ \\
\hline & PSS-C & & $-.24 * *$ & PSS-C & & $-.17 *$ \\
\hline \multirow[t]{5}{*}{ Step 3} & & $.21 * *$ & & & $.29 * *$ & \\
\hline & Sex & & .04 & Sex & & .05 \\
\hline & LS-G & & -.21 & LS-S & & .28 \\
\hline & PSS-C & & -.71 & PSS-C & & $-.28 *$ \\
\hline & LS-G x PSS-C & & $.68 * *$ & LS-S x PSS-C & & .27 \\
\hline Total $R^{2}$ & & & & & & \\
\hline
\end{tabular}

Note. LS-G: general-life stress ; LS-S: sport-specific life stress ; PSS-C: PSS counter stress; $* p<.05, * * p<.01$. 


\section{Figure 1 (on next page)}

Relationship between two types of life stress and burnout moderated by counter stress

Relationship between two types of life stress and burnout moderated by counter stress 


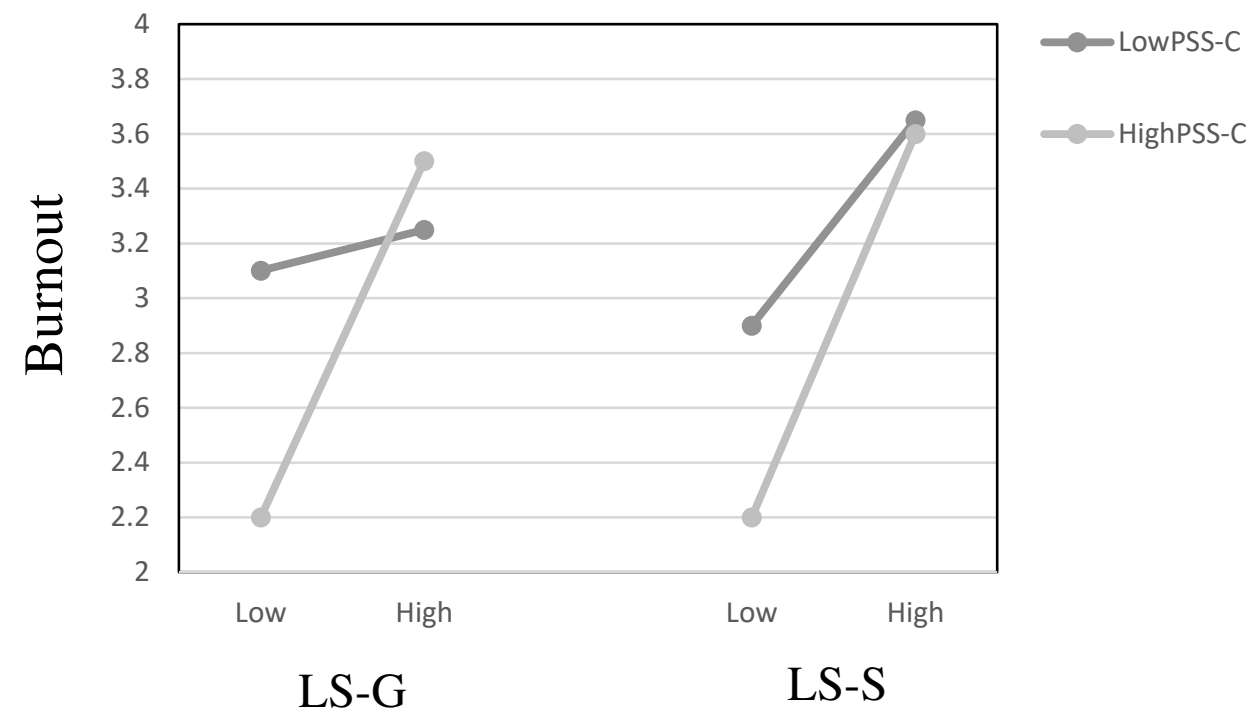

Figure 1. Relationship between two types of life stress and burnout moderated by counter stress.

Note: ${ }^{a}$ For participants with high counter stress (light line), burnout was significantly lower than participants with low counter stress in general-life stress condition but not in sport-specific life stress condition.

${ }^{\mathrm{b}}$ LS-G: general-life stress ; ${ }^{\mathrm{c}} \mathrm{LS}-\mathrm{S}$ : sport-specific life stress 